\title{
APPLICATION OF POWER LINE COMMUNICATION TECHNOLOGY IN STREET LIGHTING CONTROL
}

\author{
TEKLA D. FARKAS ${ }^{1}$, TAMÁS KIRÁLY ${ }^{2}$, TAMÁS PARDY ${ }^{1}$, TOOMAS RANG ${ }^{1}$ \& GALINA RANG \\ ${ }^{1}$ Thomas Johann Seebeck Department of Electronics, Tallinn University of Technology, Estonia. \\ ${ }^{2}$ Flashnet Hungary Kft, Hungary.
}

\begin{abstract}
Rapidly increasing usage of telecommunication systems causes new transmission technologies and networks to emerge. Not only the efficiency, reliability and accessibility of the network are important, but also the economic issues. One cost-effective solution could be power line communication (PLC) technology, which transmits data using the existing electricity infrastructure. The application of this communication technique is an attractive and innovative solution for the realization of smart cities and smart homes. With intelligent control networks, energy savings can be optimized and the operating as well as maintenance costs can be reduced. Since outdoor lighting systems are the major consumers of electricity, to create a modern, energy-efficient city, intelligent street lighting control is needed. This paper provides an overview of power line communication principles including the theoretical background of data communication, modulation techniques, channel access methods, protocols, disturbances and noises. Furthermore, in order to highlight the benefits of a PLC-based street lighting control system, a pilot project will be presented.
\end{abstract}

Keywords: intelligent lighting control, modulation techniques, Power line communication, smart city, smart grid.

\section{INTRODUCTION}

Power line communication (PLC) is a very attractive technology, which can be used for many applications, such as home automation systems, CCTV cameras, pollution sensors, noise detectors, traffic density sensors, advertising backlit panels, solar applications, energy measurements, street lighting control or vehicle communication networks [1].

Energy usage of street lighting accounts for a significant portion of the total energy consumption of a city [2]. In order to reduce energy consumption, $\mathrm{CO} 2$ emission, light pollution, to improve public safety and enhance public service quality, implementation of an intelligent light control system is essential [1]. PLC is a natural choice for supporting intelligent lighting control systems in order to ensure the optimal illumination level under any operational circumstances and to reduce operational costs.

A PLC network can also be the backbone communication infrastructure for a smart grid, which enables reliable, real-time measurements, monitoring and control [3]. The implementation of this management solution is cost-effective, since the communication is done over the existing power lines and therefore no additional infrastructure is needed. Due to this fact, PLC technology can compete with wireless technology and can be a communication medium in a future smart grid [4].

There are three basic network elements for the realization of a PLC system: base station, modem and repeater. PLC technology provides a link between the subscribers and their immediate service provider. The application of PLC in the power supply grid can be considered as a communication solution for the access network. To connect the access network to a wide area network (WAN), a PLC base station is used. To make communication possible over power supply grids, PLC modems are needed between the communication devices and power lines, which convert data signals into a suitable form for both directions of transmission. A PLC modem has two interfaces: on the user side, it provides Ethernet and USB network 
interfaces and in order to connect the PLC modem to the power line, PLC signal injection should be realized by inductive or conductive coupling. The PLC modem carries out the function of the physical layer (PHY) and the data-link layer (DLL, including both of the sublayers, the media access control (MAC) and the logical link control (LLC)). In case of longer network distances, repeaters are used to ensure sufficient data rate. The repeaters divide the PLC access network into network segments. In order to separate the segments, different frequency bands or time slots are used. With segmentation, signal power and electromagnetic radiation can be reduced, but network capacity decreases, because wider frequency bands have to be used to avoid the interference between the segments $[5,6]$.

Gateways are usually used for better network efficiency, dividing the PLC access network into subnetworks. Gateways not only forward the data between the networks, but can also interconnect networks, which use different network protocols by performing the required protocol conversions. The drawback of this setup is that repeaters and gateways are expensive elements and because of the signal conversion, propagation delay can occur. It is also of note that the frequency ranges are limited in the case of PLC, and losses on the medium can negatively influence power transmission. Various noise sources (different electrical devices connected to the power supply grids with the same electromagnetic spectrum) can also cause disturbances in the PLC service [5, 6]. In this paper, we provide a short overview about the theory, principles and basic features of PLC systems. Furthermore, to highlight the attractiveness of this technology, a PLC based street lighting control system will be introduced.

\section{THEORETICAL BACKGROUND AND BASIC FEATURES OF PLC SYSTEMS}

\subsection{Frequency ranges for PLC}

There are two frequency ranges for PLC: narrowband and broadband. Defined by CENELEC, narrowband PLC (NB-PLC) networks operate between 3-148.5 kHz in Europe. This frequency range is divided into four bands: $\mathrm{A}, \mathrm{B}, \mathrm{C}$ and $\mathrm{D}$. Band $\mathrm{A}[3-95 \mathrm{kHz}]$ is restricted to the energy suppliers, frequency bands B [95-125 kHz], C [125-140 kHz], D [140-148.5 kHz] can only be used by consumers. Typically, NB-PLC systems operate between $35-90 \mathrm{kHz}$ in the CENELEC A band, while broadband PLC (BB-PLC) networks use the 3-34 MHz frequency band [5, 7].

The NB-PLC systems can provide data rates up to few thousand bps, while the BB-PLC systems have reached few hundred Mbps [8]. These higher data rates can enable high-speed data transmission, multiple voice connections and transfer of video signals as well. The main differences between narrowband and broadband PLC are in frequency range, information capability, power and attenuation $[5,7]$.

\subsection{Modulation techniques}

Modulation techniques are methods, which are used to code analogue data into digital format. There are various types of digital modulation schemes depending on the type of the signal and the application [9]. The most common types will be described below.

1. Amplitude Shift Keying (ASK): in this case, the carrier signal is modulated with a binary message ( 0 or 1$)$. The carrier signal is transmitted, if the signal value is 1 , but when this value is 0 , the carrier signal will not be transmitted. The amplitude shift keying (ASK) technique is sensitive to disturbances and it has not constant envelope, 
which makes power amplification more difficult. The first NB-PLC systems were realized by this modulation technique $[5,10]$.

2. Frequency Shift Keying (FSK), Binary Frequency Shift Keying (BFSK): digital information is transmitted through discrete frequency changes of the carrier wave. The two different digital values of 0 and 1 represent two different frequencies [10]. This modulation technique can be used for narrowband and broadband communications depending on the separation of the two frequencies [5]. Although it is a cost-effective solution, it is not bandwidth-efficient and requires a complex receiver design.

3. Phase Shift Keying (PSK), Binary Phase Shift Keying (BPSK), Quadrature Phase Shift Keying (QPSK): when the carrier wave phase is discretely varied, the modulation scheme is called phase shift keying (PSK). In the case of BPSK, two different digital values $(0$ and 1$)$ are used and when a binary change occurs in the serial binary data, the carrier wave shifts $180^{\circ}$. For QPSK, bit pairs are used. Each bit pair represents different carrier phase shifts. The bit pairs and phase degrees are the following: $00-45^{\circ}, 01-135^{\circ}$, $11-225^{\circ}, 10-315^{\circ}$, where for instance a phase change of $45^{\circ}$ means 00 . The amount of the information associates with a phase or the number of the discrete phases that the carrier wave contains. In case of ASK, BFSK and BPSK, the baud rate is $1 \mathrm{bit} / \mathrm{symbol}$, while QPSK can transmit 2 bits/symbol [11, 12].

4. Quadrature Amplitude Modulation (QAM): quadrature amplitude modulation (QAM) is the combination of amplitude and phase modulation. For instance 16-QAM uses twelve carrier phases plus three amplitude levels to transmit 4 bits per symbol [11, 12].

5. Orthogonal Frequency Division Multiplexing (OFDM): orthogonal frequency division multiplexing (OFDM) is a multicarrier modulation technique. In contrast with singlecarrier, the data is not transmitted by only one carrier, but they are shared among several carriers and are transmitted simultaneously. The single high-rate data stream is subdivided into N low-rate parallel substreams. Each substream is modulated over a separate carrier signal subcarrier, moreover each subcarrier can be modulated differently (BPSK, QPSK etc.). The subcarriers are not separated but overlapped and they are orthogonal, which means that the peak of one subcarrier occurs when other subcarriers are zero [5, $13,14]$. OFDM has several advantages. It is robust in a multipath propagation environment, more resistant against fading, tolerant to delay spread and it can minimize the effect of intersymbol interference (ISI) [5]. Since N different subcarriers are used, the symbol duration of a signal is $\mathrm{N}$ times longer than the signal duration of the original signal. To avoid interference, a guard interval (cyclic prefix) is used between the signals. The cyclic prefix is a copied portion of the end of a symbol, which is put at the head of the symbol.

\subsection{Spread-spectrum techniques}

The spread-spectrum method is a technique in which a pseudo noise code, independent from information data, is used as a modulation waveform. It results in greater bandwidth than the signal information bandwidth. The big advantage of these techniques is that the broadband, noise-like signals are more resistant to interference, noise and jamming, and it is harder to detect, intercept and demodulate them than narrowband signals [5, 15]. Commonly used techniques are listed below:

1. Direct Sequence Spread Spectrum (DSSS): In this method, the binary data (bits) are logically compared to a string of pseudo noise (PN) code bits (chips). The chip rate 
$\left(R_{c}=1 / T_{c}\right)$ is much higher than the data rate $\left(R_{s}=1 / T_{s}\right)$. The binary data is multiplied with the PN code to produce the transmitter signal. The effect of the multiplication of the bits and chips is the spread of the baseband bandwidth $R_{c}$ to the baseband $R_{s}$. Afterwards, usually BPSK or PSK modulations are used to transmit the signal to the high frequency. The receiver compares the spread spectrum signal with the same chip sequence used in the transmitter and then, a demodulator is applied to reproduce the transmitted data [15].

2. Frequency Hopping Spread Spectrum (FHSS): This method spreads narrowband transmitted signals, hopping one frequency to the next. The transmission frequencies change according to the pseudo noise sequence. Compared to the above-mentioned spreadspectrum techniques, DSSS can provide higher data rate than FHSS, since in order to increase the data rate at DSSS, only the clock rate has to be increased, while at FHSS a wider bandwidth is needed. DSSS transmission is continuous and broadband, while FHSS transmission is discontinuous and narrowband. FHSS is more susceptible to narrowband noise and interference, than DSSS [5, 15].

\subsection{Channel Access Method (CAM):}

Channel access methods are used to allow individual users to use the same fixed bandwidth. There are three basic access schemes depending on which channel is separated, but their combination can also be used [13]:

1. Frequency-division multiple access (FDMA): in an FDMA scheme, the available system bandwidth is divided into non-overlapping bands. In order to reduce the interference, there is a guard band between the adjacent frequencies. The frequency bands are assigned among the users, so that no other user can use the same band. The frequency band can be reassigned, when the user terminates the service [13].

2. Time division multiple access (TDMA): TDMA allows the users to use the same band by dividing the signal into different time shots. To avoid that data from adjacent time slots interfere, there is a protection intervals (guard period) between the time slots. A certain number of timeslots forms a TDMA frame. When disturbances occur, just the erroneous, but segmented data packets have to be retransmitted, therefore this scheme is widely used in PLC technology [5, 13].

3. Code division multiple access (CDMA): the CDMA access method transmits the data according to a special coding scheme. This method enables to send information simultaneously over a single communication channel [13].

\subsection{Protocol}

In PLC, the medium is listened to by both the PHY level (physical carrier sense) and the MAC layer level (virtual carrier sense). In order to avoid collision by different users, the channel is listened to before sending the data. If the medium is free for a DCF interframe space (DIFS) interval, transmission is permitted. If the frame is successfully received, the receiver sends an acknowledgment frame (ACK) to notify the source of successful reception. The time between the reception of a frame and the transmission of the ACK is called short inter-frame space (SIFS). If the channel is used, the sender must wait a random period of time before attempting to access the medium again (back off time). In case of virtual 
carrier sense mechanism, first the sender transmits an RTS (request-to-send) packet, which indicates the transmission time required for the data and the ACK packet. Receiving the RTS packet, the destination station sends back a CTS (clear-to-send) packet after SIFS time. Implementation of the RTS/CTS mechanism can help avoid collisions, since each station in the range of the receiver is informed about the transmission [5].

\subsection{Coupling}

There are two different methods, inductive and capacitive coupling, to connect PLC devices to the transmission medium $[16,17]$. The basic function of the coupling circuit in a PLC modem ensures the maximum power for both transmission and reception modes. It protects devices from transient components and rejects noise signals. Inductive coupling is noisier than capacitive coupling, but it can reduce attenuation by 10 to $15 \mathrm{~dB}$ for some frequencies $[16,18]$. It can also be an advantage that no physical connection to the grid has to be made, because the inductive couplers are electrically insulated from the power cables. In case of inductive coupling, a ring-shaped ferrite is clamped around the cable in order to inject the communication signal. The inductive coupler can be considered a transformer, which has a modem-side primary winding, and a power cable side secondary winding. In this way, the signal is directly induced through the coupler, to and from the power cable. Capacitive coupling is the preferred method at coupling points with relatively high impedance $(>20 \Omega)$ and when inductive coupling is not possible due to high current ratings, or the geometry of the couplers cannot allow the access due to too small inner diameter of the ferrite $[16,17]$.

\subsection{Power supply grid}

Realization of the PLC network depends on the power supply grid levels. There are three power supply grid levels, high-, medium- and low-voltage level. The high-voltage grid (110 $\mathrm{kV}$ or above) is the backbone of the electricity supply system. It is a part of the transmission stage and connects power stations with large supply regions or big consumers. It enables long-distance transmission using high voltage $(110 \mathrm{kV}, 220 \mathrm{kV}, 330 \mathrm{kV}, 400 \mathrm{kV}, 750 \mathrm{kV})$ in order to reduce line losses. Power is usually transmitted through overhead power lines, but in urban areas or sensitive regions underground power transmission is used. The medium-voltage grid (usually 10-30 kV) supplies urban and rural areas. Overhead and underground power lines are also used. MV grids usually have ring topology. Low-voltage grid (400 V) supplies households and small industrial clients. Home electrical installations belong to the low-voltage level, but are owned by the users [19]. PLC systems are primarily used in medium- or low voltage grids. The attenuation is determined by the cable and coupling losses. The attenuation is lower and less frequency selective on the medium-voltage grid, than on the low-voltage grid. On the other hand, attenuation shows frequency-dependence; the coupling loss decreases with increasing frequency. From a certain frequency, the cable loss becomes dominant, therefore the attenuation increases again. An underground MV power grid is less influenced by noises, compared with LV power grid [5, 19].

\subsection{Disturbance, noises}

Power supply grids are not primarily designed for communication. The transmission channel is characterized by different types of noises, frequency-dependent attenuation, changing 
impedance and fading. The physical properties of electrical wiring influence the transmission of the data. Various electronic devices (resistive, inductive and capacitive elements) connected or disconnected to or from the grid constantly modify the inductive and capacitive characteristics of the grid. On the other hand, PLC systems produce electromagnetic radiation, which can cause disturbances in other services operating in the same frequency range [20].

\subsection{Electromagnetic compatibility (EMC)}

Electromagnetic compatibility refers to emissions and susceptibility of different systems, which use the same frequency band. The classification of EMC disturbances according to the spectrum can be seen on. In case of PLC systems, the main source of EMC is the radiated and conducted emission from the asymmetric cables in LV and MV grids [5, 21].

\subsection{Noise}

Noise can be categorized into the following classes [5]:

1. Coloured background noise: this noise is a slow, time-variant random disturbance. The power spectral density decreases with increasing frequency.

2. Narrowband noise: this noise is mainly caused by broadcasting and wireless communication systems. The radio signals of these can impair the PLC channel. Narrowband noise mostly has a sinusoidal waveform.

3. Periodic impulsive noise, asynchronous to the main frequency: this noise is mainly caused by switching power supplies. Its frequency range is from $50 \mathrm{kHz}$ to $2 \mathrm{MHz}$ with a form of impulses which has a repetition rate between $50 \mathrm{kHz}$ and $200 \mathrm{kHz}$. This type of noise results in discrete lines in the spectrum.

4. Periodic impulsive noise, synchronous to the main frequency: this type of noise is mainly caused by the power supply, which operates synchronously with the main frequency. The repetition rate of this impulse is synchronous with the frequency mains or its double.

5. Asynchronous impulsive noise: this noise is usually caused by switching transients. Duration of impulses is from a few microseconds to milliseconds with arbitrary inter-arrival times. The asynchronous impulsive noise has high power spectral density (greater than $50 \mathrm{~dB}$ ), thus this class of noise causes most PLC system errors [8].

\section{PILOT PROJECT: INTELLIGENT LIGHTING CONTROL FOR LIGHTING NETWORK SYSTEM}

\subsection{Experimental setup}

\subsubsection{Overview}

Intelligent control of street lighting systems is a vital part of smart city projects. It can offer several benefits and reliable solutions for a city: it can help save energy by dimming light sources, enhance safety by real time monitoring and analysis of the network and reduce maintenance costs by intelligent optimization [1]. In the following, a pilot project will be introduced in detail, which is operated in Pilisborosjenő, in Hungary [22]. The system architecture consists of three levels: lamp-level, remote terminal unit level and software control 
level. The control and monitor of the lamps are realized with InteliLIGHT Streetlight Management System. 27 street lighting fixtures are controlled, of which 11 are highpressure sodium lamps (4 pieces of $100 \mathrm{~W}$ light bulbs and 7 pieces of $70 \mathrm{~W}$ light bulbs), 13 are compact fluorescent lamps ( $36 \mathrm{~W}), 2$ are LED lamps (18 W, $36 \mathrm{~W})$ and 1 is a metal-halide lamp $(400 \mathrm{~W})$. Each lamp has its own ON/OFF switching and/or dimming function, providing the possibility to control them individually. The controllers are mounted on street lighting fixtures, while the communication devices (electric network analyser, data concentrator, communication router and modem) are placed inside the power supply cabinet. PLC is used between the controller and communication devices, and IP-based communication is used for data transfer between the controllers and the management software [1]. Figure 1. shows the location of the lights, including a table that contains the light bulb types and controllers. Figure 2. shows the hardware used for control and analysis.

\subsubsection{Controllers}

FPM-150 and FPM-400 (up to $150 \mathrm{~W}$ and $400 \mathrm{~W}$ respectively) type electromagnetic ballast controllers were used for HPS and MH lamps. The controllers switch the lights on/off and control the brightness setting (dimming). They are also responsible for collecting information about the system status (failures, faults etc.) and features (voltage, current etc.). FPX-220 on/

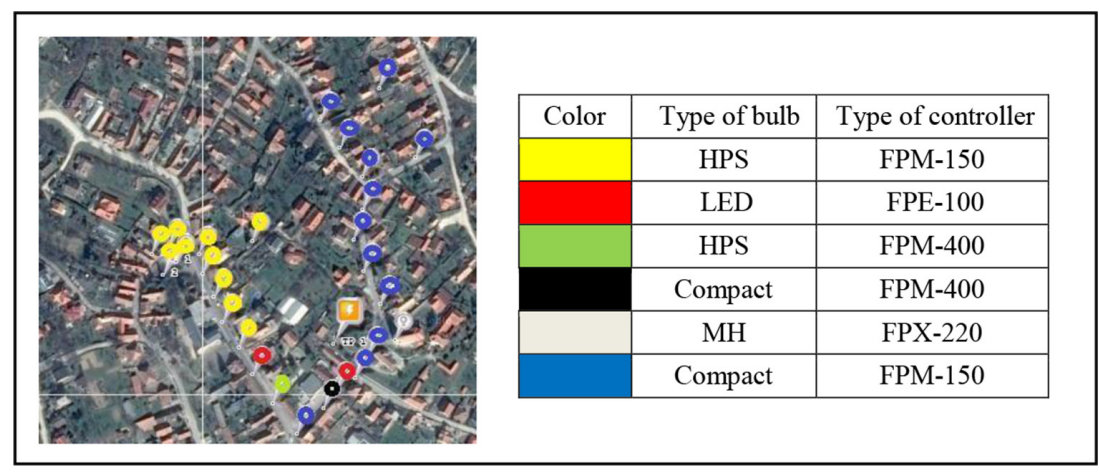

Figure 1: Locations of the lighting fixtures, types of bulbs and controllers
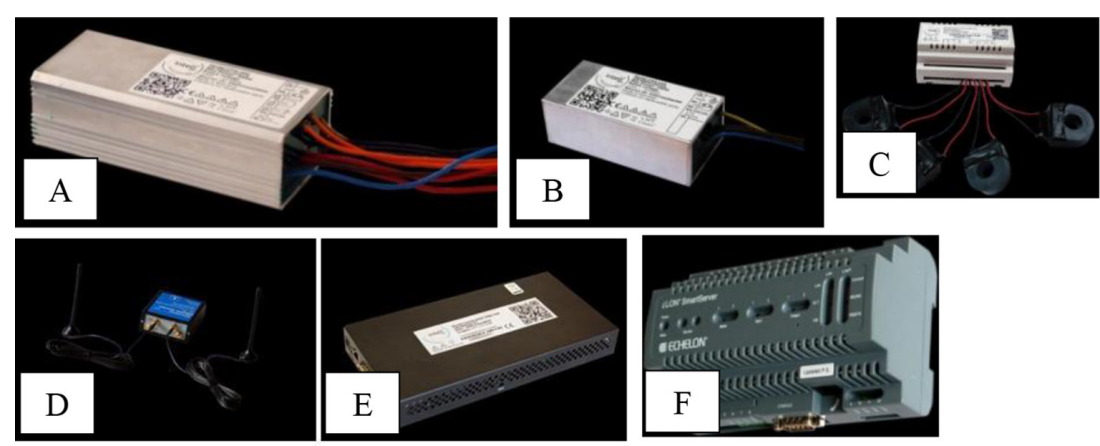

Figure 2: FPM-400 (A), FPX-220 (B), FNM-110 (C), Teltonica router and FRB-100 modem (D\&E), FPC-200 (F) 
off electronic controller can be used for any types of lamps, but it does not provide dimming control function. Since constant illumination was needed for the floodlighting of the church, FPX-220 was a good choice for the remote management of the luminaries. The controllers use the CENELEC C band and LonWorks, peer-to peer protocol. It uses a narrowband spread-spectrum modulation scheme and CSMA in order to avoid data collisions [8].

\subsubsection{Electric network analyser (FNM-110)}

Electric network analyser can enable measurements and analysis of the street lighting grid parameters. It has $\mathrm{R}, \mathrm{S}, \mathrm{T}, \mathrm{N}$ current inputs. It can send report about effective voltage and current, frequency, active, reactive and apparent power and power factor.

\subsubsection{Data concentrator (FPC-200)}

FPC-200 data concentrator is a software interface for configuration, programming, system integration and failure alarming. It receives the data from the components and converts the information using LonWorks protocol. After converting the data, the information packages are sent to the central processing unit.

\subsubsection{Communication router (Teltonica, RUT 104) and modem (FRB-100)}

Communication router provides VPN connection between controllers, network analysers, data concentrators and InteliLIGHT Control Software. The router saves data logs and history of the events on the grid and archives them. The FRB100 modem saves and archives data logs and history of the events on the grid and ensures the secure data transmission to the Network Management Software.

\section{RESULTS AND DISCUSSION}

The lighting fixtures are situated on two different lighting circuits (Phase 1, Phase 3). On Phase 1, mainly high-pressure sodium (HPS) lamps are situated, and ON/OFF electronic controllers are used, while on Phase 3, mainly compact fluorescent lamps are situated, and dimming control is used (see Fig. 1.). Streetlights are controlled according to a predefined schedule, which is based on the experience and demand of the local government and energy supplier. Streetlights are switched ON/OFF at 20 minutes before/after the sunset/sunrise, and the controlling is done at $11 \mathrm{pm}$ and $12 \mathrm{pm}$ to $90 \%$ and $80 \%$ of the nominal power. To estimate how the active and reactive power can be changed by the control, a one-month long period (01-30.09.2016.) was investigated. On Fig. 3. A, B, the change of the instantaneous and total active power can be seen (only a one-week period is depicted). Since on Phase 1 only ON/OFF electronic controllers are used, the active power was constant $(\sim 827 \mathrm{~W})$. In case of Phase 3, where the dimming function is used, the active power was $\sim 1293 \mathrm{~W}$ before the control. In the first and second steps of the control process, the value of the active power was $\sim 1238 \mathrm{~W}$ and $\sim 1169 \mathrm{~W}$, respectively. This means that the active power decreased with $\sim 9.6 \%$ in total; $\sim 4.2 \%$ in the first step and $\sim 5.4 \%$ in the second step. Table 1 summarizes active power values for phase $1 \& 3$.

On Fig. 3. C, D, the change of the instantaneous and total reactive power can be seen for the period of 16.09.2016-23.09.2016. In case of Phase 1, the reactive power was almost constant ( $661 \mathrm{VAr}$ ). In case of Phase 3 no significant difference can be measured; before the control, the value of the reactive power was $1330 \mathrm{VAr}$ which decreased to $\sim 1328 \mathrm{VAr}$ and $\sim 1296$ VAr, respectively ( $\sim 3 \%$ in total). Table 2 summarizes reactive power values for phase $1 \& 3$. 

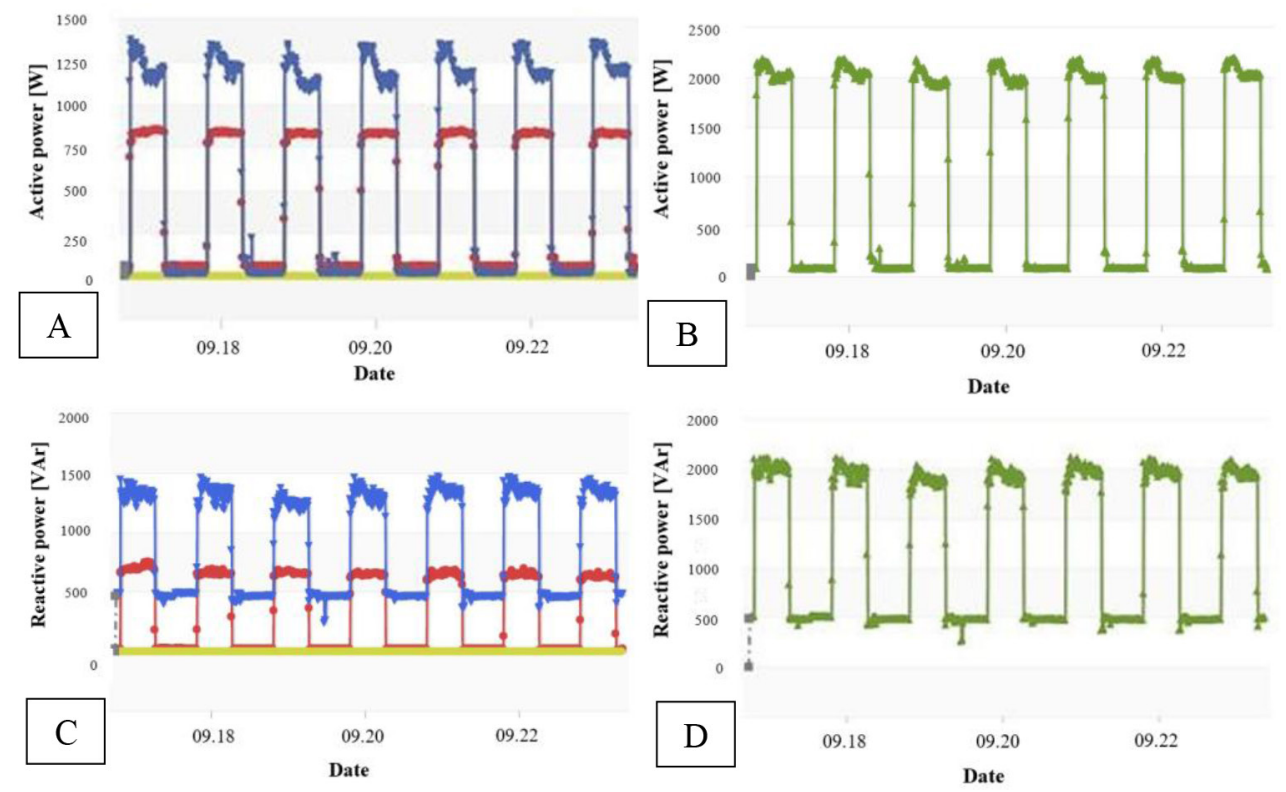

Figure 3: Instantaneous active power (A), Total instantaneous active power (B), Instantaneous reactive power $(\mathrm{C})$, Total instantaneous reactive power $(\mathrm{D})$. Plot colours: red:=Phase 1 , blue:=Phase 3 .

Table 1: Active power values for Phase $1 \& 3$.

\begin{tabular}{lll}
\hline & $\begin{array}{l}\text { Phase 1 (HPS lamp }+ \\
\text { ON/OFF control) }\end{array}$ & $\begin{array}{l}\text { Phase 3 (fluorescent lamp + } \\
\text { dimming control) }\end{array}$ \\
\hline Active power (no control) & $\sim 827 \mathrm{~W}$ & $\sim 1293 \mathrm{~W}$ \\
Active power (step 1) & $\sim 827 \mathrm{~W}$ & $\sim 1238 \mathrm{~W}$ \\
Active power (step 2) & $\sim 827 \mathrm{~W}$ & $\sim 1169 \mathrm{~W}$ \\
\hline
\end{tabular}

Table 2: Reactive power values for Phase $1 \& 3$.

\begin{tabular}{lll}
\hline & $\begin{array}{l}\text { Phase 1 (HPS lamp }+ \\
\text { ON/OFF control) }\end{array}$ & $\begin{array}{l}\text { Phase 3 (fluorescent lamp }+ \\
\text { dimming control) }\end{array}$ \\
\hline Reactive power (no control) & $\sim 661 \mathrm{VAr}$ & $\sim 1330 \mathrm{VAr}$ \\
Reactive power (step 1) & $\sim 661 \mathrm{VAr}$ & $\sim 1328 \mathrm{VAr}$ \\
Reactive power (step 2) & $\sim 661 \mathrm{VAr}$ & $\sim 1296 \mathrm{VAr}$ \\
\hline
\end{tabular}

These results are in good agreement with the purposes of this study: with intelligent lighting control system, energy consumption can be decreased. It should also be noted that the quality of the power supply grid, such as age and material of the cables, types of cable shielding and insulation, corrosion of cable joints, substantially affects the propagation of the signal. Away from the power supply cabinet the signal strength can drop and the probability and duration of the outage can increase. 


\section{CONCLUSIONS}

In this paper, we reviewed the theoretical background of PLC technology related to smart street lighting control, including frequency ranges, modulation techniques, protocols as well as coupling methods and the characteristic power supply grid. Furthermore, a pilot project demonstrating the benefits of intelligent lighting systems was detailed. The project was carried out in a small town of about 3300 inhabitants with 27 lamps monitored for power consumption as well as controlled through PLC. The pilot study concluded that an up to $\sim 9.6 \%$ decrease of active power was achievable by smart lighting control. PLC technology is a low-cost, environment-friendly and flexible communication technology, which is an ideal candidate for the realization of smart grid networks. Future works will be targeted at extending the project to more heavily populated areas, namely cities at least one order of magnitude larger in population. The larger the settlement, the more complex the power grid as well as the more challenging it is to maximise power efficiency while causing minimal disruption to night activities characteristic of larger settlements.

\section{REFERENCES}

[1] InteliLIGHT® street lighting management system, FlashNet Srl, available at https:// intelilight.eu/ (accessed 25 September 2016).

[2] How much electricity is used for lighting in the United States?, U.S. Energy Information Administration, available at https://www.eia.gov/tools/faqs/faq.php?id=99\&t=3 (accessed 25 September 2016).

[3] Municipality use case, Echelon, available at http://www.echelon.com/assets/blt65a02a03328f66e8/Municipality\%20Use\%20Case.pdf

[4] Caytiles, R.D. \& Lee, S., A survey of recent power line communication technologies for smart micro grid. International Journal of Software Engineering and Its Applications, 9(12), pp. 251-258, 2015. https://doi.org/10.14257/ijseia.2015.9.12.22

[5] Hrasnica, H., Haidine, A. \& Lehnert, R., Broadband Powerline Communications: Network Design, Chichester, John Wiley \& Sons, 2005.

[6] Vörös, B., BPL technológia, Master Thesis, Budapest University of Technology and Economics, 2012.

[7] Powerline Communications Emerge as Core Networking Technology, Electronic Design, available at http://www.electronicdesign.com/energy/power-linecommunicationsemerge-core-networking-technology (accessed 25 September 2016).

[8] Yigit, M., Gungor, V.C., Tuna, G., Rangoussi, M. \& Fadel, E., Power line communication technologies for smart grid applications: a review of advances and challenges. Computer Networks, 70, pp. 366-383, 2014. https://doi.org/10.1016/j.comnet.2014.06.005

[9] Sharma, D. K., Mishra, A. \& Saxena, R., Analog \& Digital Modulation Techniques: An overview. International Journal of Computing Science and Communication Technologies, 3(1), p. 2007, 2010.

[10] Amplitude Shift Keying \& Frequency Shift Keying, available at http://www.ele.uri.edu/ Courses/ele436/labs/ASKnFSK.pdf (accessed 25 September 2016)

[11] Understanding Modern Digital Modulation Techniques, Electronic Design, available at http://electronicdesign.com/communications/understanding-modern-digital-modulation-techniques (accessed 25 September 2016) 
[12] What's the Difference Between Bit Rate and Baud Rate, Electronic Design, available at http://electronicdesign.com/communications/what-sdifference-between-bit-rate-andbaud-rate (accessed 25 September 2016)

[13] Third Generation Wireless Networks, Sky DSP, available at http://www.skydsp.com/ publications/4thyrthesis/chapter1.htm

[14] Xiao, Y., Orthogonal frequency division multiplexing modulation and inter-carrier interference cancellation, 2003.

[15] Meel, J., Spread spectrum (SS), Nayer Inst. Hogesch. Voor Wet. Kunst, 1999.

[16] Carcelle, X., Power Line Communications in Practice, New York, Artech House, 2009.

[17] Ferreira, H.C., Grové, H.M., Hooijen, O. \& Vinck, A.H., Power Line Communication. Wiley Online Library, 2010.

[18] Siong, A.K., Design and Development of a Power Line Communication System, The University of Newcastle, Australia, 2010.

[19] Berger, L.T., Schwager, A. \& Escudero-Garzás, J.J., Power line communications for smart grid applications. Journal of Electrical and Computer Engineering, 2013, p. 3, 2013.

https://doi.org/10.1155/2013/712376

[20] Papadopoulos, T.A., Kaloudas, C.G., Chrysochos, A.I. \& Papagiannis, G.K., Application of narrowband power-line communication in medium-voltage smart distribution grids. IEEE Transactions on Power Delivery, 28(2), pp. 981-988, 2013. https://doi.org/10.1109/tpwrd.2012.2230344

[21] Vukicevic, A., Electromagnetic Compatibility of Power Line Communication Systems, Switzerland, Lausanne, 2008.

[22] Bottyán, B., Király, T., Smart technológia alapú közvilágítás, 60. MEE Vándorgyülés, ID 5347, 2013. 